International Journal of Engineering \& Technology, $9(1)(2020) 37-51$
International Journal of Engineering \& Technology
SPC
Website: www.sciencepubco.com/index.php/IJET
Research paper

\title{
Design and analysis of attitude control algorithm for low earth orbiting satellite with magnetic torquer concepts using nonlinear unscented Kalman filter
}

\author{
M. Raja ${ }^{1}$, Dr Ugur Guven ${ }^{2}$, Dr Om Prakash ${ }^{2}$ \\ ${ }^{1}$ Assistant Professor, Department of Aerospace Engineering, University of Petroleum and Energy Studies, Dehradun-248007, \\ Uttarakhand, India \\ ${ }^{2}$ Professor, Department of Aerospace Engineering, University of Petroleum and Energy Studies, Dehradun-248007, \\ Uttarakhand, India \\ *Corresponding author E-mail: mraja@ddn.upes.ac.in
}

\begin{abstract}
Attitude control system plays the important role for to maintain the satellite to desired orientation. To control the satellite it is necessary to do the attitude stabilization. Attitude stabilization achieved by Star sensor, sun sensor, Earth sensors. Attitude control is mainly use for antenna pointing accuracy, camera focus to earth surface and solar panel pointing toward sun. Due to tumbling effects, satellite will rotate all the direction in the space. To maintain the orientation of the satellite it is necessary to design the attitude determination and control. Satellite consider as the rigid body. Inertia matrix describes the rigid body dynamics. The orientation of the satellite determine by Euler angle and Quaternion. The Low earth orbiting satellite will have enormous amount of aerodynamic drag stinking the satellite body and gravitational attraction another problem. Because of that satellite dwell, time is reduced. It means satellite more time spending particular part of the earth. The attitude estimation is measures by the orientation of vectors. Attitude estimation means to find the position and orientation of flying object with respect to the fixed reference of reference. Vector remains considered in the frame of reference to compute for find the orientation of the body of the satellite in the inertial reference system. The Earth is an inertial reference frame, and Satellite is a body frame. Attitude sensor used to measure the satellite orientation in the reference frame. This will help in accurately predicting the orbit deviation and a control system to correct if any by providing the satellite momentum means 'mass in motion' changes in a body rapidly in Low earth orbit due to centripetal force acting on a satellite. Attitude control system (ACS) need the numerical simulation to find the required torque demand by the help of difference between reference input (Attitude) signal and feedback signal measure by attitude sensor to trim the control surface maintain the actuator required orientation. The results will consist of two parts the first part consisting of the attitude estimation using Euler angle and Quaternion method, second part consist of estimate the control torque from magnetic torquer and error estimation using non-linear filter (Unscented Kalman Filter) with MATLAB simulation.
\end{abstract}

Keywords: Attitude and Determination and Control System (ADCS); Coordinate Frame; Direct Cosine Matrix; Magnetic Torquer; Unscented Kalman Filter.

\section{Introduction}

The spacecraft attitude control is very important to stabilize the satellite along with orientation. The perturbation forces (or) environmental disturbances affects the satellite from original orbit to perturbed orbit. It is very important to reduce the perturbation forces. To maintain the satellite pointing accuracy Such as Communication antenna pointing towards earth surface, Solar panel pointing towards Sun. Because thruster require energy from sun to re-orient the satellite to the desired path. Example: Remote sensing satellite to capture the earth surface it require the camera should be pointing towards earth. It is require attitude-stabilizing technique to maintain the required path. Environmental force also differs with different altitude. For, Low earth orbit satellite will affect more aerodynamic drag and gravitational attraction due to earth, solar flux, Earth magnetic field due to non-spherical earth surface, J2 Perturbation. [1] High earth orbit have three body problems such as moon attraction. Spacecraft stabilization classifies two methods Spin stabilization and Gravity gradient. Spin stabilization need energy to control the satellite. Actuators is use to reorient the satellite in the desire path. Control momentum gyroscope (CMG) measure the disturbance torque. This Feedback signal is to trigger the satellite to move the control surface. CMG might be single gimbal or Dual Gimbal. The momentum exchange device such as Reaction wheel also used to stabilize the satellite attitude control. The magnetic thruster it is a dipole interacts with the earth magnetic flux to generate the control torque to maintain its specific path especially for Low earth orbit satellite. Passive stabilization does not require any external power supply. [2] 


\subsection{The earth-satellite system}

The simplest of the n-body problems is the two-body problem, only two masses are consider at a time. Let the masses be consider by m1 and $\mathrm{m} 2$. The n-body equation becomes

$$
\begin{gathered}
\mathrm{F}_{1}=\mathrm{m}_{1} \mathrm{r}_{1}=G \mathrm{~m}_{1} \mathrm{~m}_{2} \frac{\mathrm{r}_{2}-\mathrm{r}_{1}}{\left|\mathrm{r}_{2}-\mathrm{r}_{1}\right| 3} \\
\mathrm{~F}_{2}=\mathrm{m}_{2} \mathrm{r}_{2}=\mathrm{Gm}_{1} \mathrm{~m}_{2} \frac{\mathrm{r}_{1}-\mathrm{r}_{2}}{\left|\mathrm{r}_{1}-\mathrm{r}_{2}\right| 3}
\end{gathered}
$$

Combining the equations (1) \& (2) gives:

$\mathrm{F}_{1}=\mathrm{m}_{1} \mathrm{r}_{1}=\mathrm{Gm}_{1} \mathrm{~m}_{2} \frac{\mathrm{r}_{2}-\mathrm{r}_{1}}{\left|\mathrm{r}_{2}-\mathrm{r}_{1}\right| 3}$

And with $r=r_{2}-r_{1}$,

$\ddot{r_{2}}-\ddot{r_{1}}=-G\left(m_{1}+m_{2}\right.$

Equation (4) is the basic equation of motion for the two-body problem. [3]

\subsection{The general n-body problem}

In a system consisting of $\mathrm{n}$ bodies, the sum of forces acting on the $\mathrm{i}^{\text {th }}$ body as shown in Equation (5)

$$
F_{i}=G \sum_{j=1}^{j=n} \frac{m_{i} m_{j}}{r_{i j}^{3}}\left(r_{j-} r_{i}\right), i \neq j
$$

It follows from Newton's second law of motion, equation that [3]

$$
\frac{d^{2} r}{d x^{2}}=G \sum_{j=1}^{j=n} \frac{m_{i}}{r_{i j}^{3}}\left(r_{j-} r_{i}\right), i \neq j
$$

\subsection{The three-body problem}

The restricted three-body problem is a very good way to describe forces between Earth, the Moon and a satellite. It consists of a system that includes three masses moving in a plane. Let us assume that Earth have mass $\mathrm{m}_{1}$, the Moon mass m2 and the satellite mass m3. Mass $\mathrm{m}_{3}$ is a lot smaller than $\mathrm{m}_{1}$ and $\mathrm{m}_{2}$, so it can neglected. The law of gravitation gives gravity force $\mathrm{F}^{\sim 1}$ on Earth from the Moon and gravity force $\mathrm{F}^{\sim 2}$ the opposite way. It has given by Equation (7)

$$
\mathrm{F}_{1}=-\mathrm{F}_{2}=\mathrm{k}^{2} \frac{\mathrm{m}_{1} \mathrm{~m}_{2}}{\mathrm{~L}^{2}} \mathrm{~b}_{1}
$$

Where $\mathrm{k}$ is the Gaussian constant of gravitation and $\mathrm{L}$ is the distance between body 1 and 2 . The vector from the center of Earth to the center of moon rotates with an angular velocity $\omega=\omega_{b 3}$ Earth has position $R_{1}=-x_{1} b_{1}$ and $R_{2}=-x_{2} b_{2}$ the Moon has Position $L$ Vector is given by $\mathrm{L}=\mathrm{x} 1+\mathrm{x} 2$. The acceleration become

$$
\begin{aligned}
& \ddot{a}_{1}=\omega X\left(\vec{\omega} \times \vec{R}_{1}\right)=\omega^{2} x_{1} \overrightarrow{b_{1}} \\
& \ddot{a}_{2}=\omega X\left(\vec{\omega} \times \vec{R}_{2}\right)=\omega^{2} x_{2} \overrightarrow{b_{1}}
\end{aligned}
$$

The gravitational and centrifugal forces are in balance. This gives

$$
\mathrm{k}^{2} \frac{\mathrm{m}_{1} \mathrm{~m}_{2}}{\mathrm{~L}^{2}}=\mathrm{m}_{1} \mathrm{x}_{1} \omega^{2}=\mathrm{m}_{2} \mathrm{x}_{2} \omega^{2}
$$

Moreover, from this Kepler's third law is found as

$$
\omega^{2}=\frac{\mathrm{k}^{2} \mathrm{M}}{\mathrm{L}^{3}}
$$

Where $M=m 1+m 2$. The position of the satellite is

$$
r=x \overrightarrow{b_{1}}=y \vec{b}_{2}
$$

The velocity is

$$
\vec{v}=\frac{d \vec{r}}{d t}+\vec{\omega}_{i b} X \vec{r}=\dot{x} \overrightarrow{b_{1}}+\dot{y} b_{2}+\omega\left(x \overrightarrow{b_{2}}-y \overrightarrow{b_{1}}\right)
$$

Moreover, the acceleration becomes 


$$
\vec{a}=\frac{d^{2}}{d^{2} t} \vec{r}+2 \overrightarrow{\omega_{1 b}} X \frac{d}{d t} \vec{r}+\vec{\alpha}_{i b} X \vec{r}+\overrightarrow{\omega_{l b}} X\left(\overrightarrow{\omega_{l b}} X \vec{r}\right)
$$

The motion of the satellite can be described as

$$
\overrightarrow{F_{3}}=-k^{2} \frac{m_{1} m_{3}}{r_{1}{ }^{3}}\left\lfloor\left(x+x_{1}\right) \overrightarrow{b_{1}}+y \overrightarrow{b_{2}}\right\rfloor-k^{2} \frac{m_{2} m_{3}}{r_{2}{ }^{3}}\left\lfloor\left(x+x_{2}\right) \overrightarrow{b_{1}}+y \overrightarrow{b_{2}}\right\rfloor,
$$

Where,

$$
\overrightarrow{\mathrm{r}_{1}}=\sqrt{\left(\mathrm{x}+\mathrm{x}_{1}\right)^{2}+\mathrm{y}^{2}}, \overrightarrow{\mathrm{r}_{2}}=\sqrt{\left(\mathrm{x}+\mathrm{x}_{2}\right)^{2}+\mathrm{y}^{2}}
$$

In the $\mathrm{x}$ and $\mathrm{y}$ direction this results in

$$
\begin{gathered}
\ddot{x}-2 \omega \dot{y}-\omega^{2} x=-k^{2}\left[\frac{m_{1}}{r_{1}{ }^{3}}\left(\left(x+x_{1}\right)+\frac{m_{2}}{r^{3}{ }_{2}}\left(x-x_{2}\right)\right]\right. \\
\ddot{y}+2 \omega \dot{x}-\omega^{2} y=-k^{2}\left(\frac{m_{1}}{r_{1}{ }^{3}}+\frac{m_{2}}{r_{2}{ }^{3}}\right)
\end{gathered}
$$

The model is usually presented in normalized form where the distances are divided by $\mathrm{L}$ and $\tau=\omega \mathrm{t}$.

\section{Motion equation of satellite $[\mathrm{EOM}]$}

We consider the mass $\mathrm{m} 1$ referred as earth mass and $\mathrm{m} 2$ satellite mass, Newton law of gravitation states that magnitude of mass directly proportional to the force of attraction and inversely proportional to the square of distance between two masses [1].

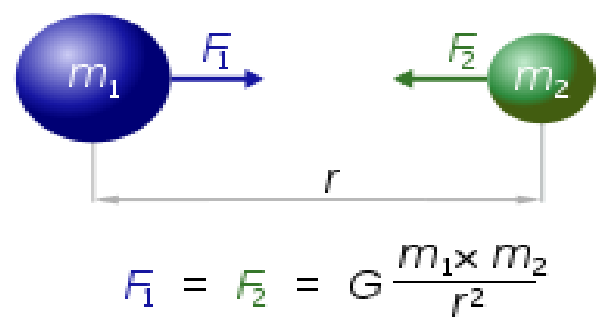

Fig. 1: Gravitation Effects.

Where: [1]

Gravitational force between two masses

- $\quad \mathrm{F} 1=\mathrm{F} 2=\mathrm{F}$ is the force of attraction between the masses, $\mathrm{N}$

- $\mathrm{G}$ is the gravitational parameter (constant), $\left(\mathrm{G}=6.674 \times 10^{-11} \mathrm{~N} \mathrm{~m}^{2} \mathrm{~kg}^{-2}\right)$

- $\mathrm{m}_{1}$ is the consider as the Earth mass, first mass, $\mathrm{Kg}$

- $\mathrm{m}_{2}$ is the consider as the flying object or satellite, second mass, $\mathrm{Kg}$

- $r$ is the line connecting the center of earth surface to center of satellite, $\mathrm{m}$

\subsection{Locating the satellite in orbit}

To find the position vector of the satellite with respect to perigee [1]

$$
\mathrm{r}_{\text {initial }}=\frac{\mathrm{a}\left(1-\mathrm{ecc}{ }^{2}\right)}{1+\mathrm{ecc} * \cos \mathrm{T}_{\text {anomaly }}}
$$

Where:

- $\quad$ ecc: eccentricity of the orbit

- a: line connecting from apogee and perigee ( Semi-major axis)

- rinitial: radius from the foci of the planet

- $T_{\text {anomaly }}$ : True anomaly (measure of the angle from the perigee to the position of the satellite

\subsection{Calculate the flight path angle and the velocity of the spacecraft by the following relations}

$$
\begin{gathered}
\text { Flight path angle }=\arctan \left(\frac{e c c * \sin T_{\text {anomaly }}}{1+e c c * \cos T_{\text {anomaly }}}\right) \\
\text { Velocity }=\sqrt{G * M\left(\frac{2}{r}-\frac{1}{a}\right)}
\end{gathered}
$$




\subsection{Energy equation for satellite}

The Energy equation of the satellite is calculates by difference between potential energy and kinetic energy. [1] Mass of the satellite, $\mathrm{m}_{\mathrm{s}}$ satellite velocity V, Semi-major axis, a[2]

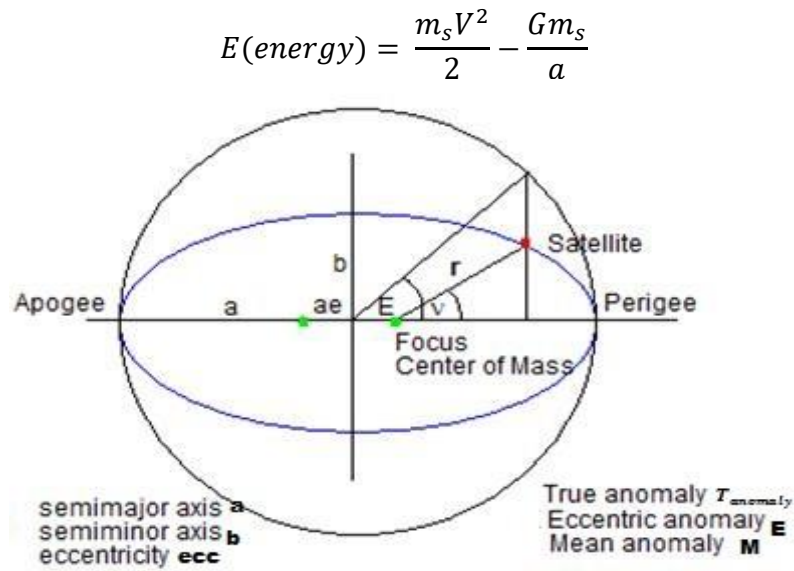

Fig. 2: Major- Minor Axis Along with Satellite Position in the Orbit.

\subsection{Circular velocity of an orbit around an object is defined as}

$$
V_{\text {circular }}=\sqrt{\frac{K^{2}}{r_{\text {earth }}}}=\sqrt{\frac{G * M}{r_{\text {earth }}}}
$$

Where for Earth

$\mathrm{K}^{2}=$ Gravitation constant $*$ Mass of the earth $=3.98 * 10^{14} \mathrm{~m}^{3} / \mathrm{s}^{2}$

Radius of the earth $=6.37 * 10^{6} \mathrm{~m}$

Hence, for escape from earth into circular orbit you would need a velocity of $7.9 \mathrm{~km} / \mathrm{sec}$

\subsection{Period calculation of a satellite}

The most simple equation for the period of a satellite is given by:

$$
T=\left(2 \Pi r_{e a r t h} \frac{3}{2}\right) /(\mathrm{K})
$$

Where $\mathrm{k}=1.9965 * 10^{7}$

\subsection{Satellite orbit elements}

Satellite orbital elements are describing the position of the satellite. North American Aerospace Defense, NORAD will provide the Two Line Elements of satellite. Such as Eccentricity, Inclination of satellite, Longitude of ascending node, Argument of perigee, Epoch, True anomaly, Semi-major axis.

\section{Orientation of the satellite}

Coordinate system is use to find the position of the object or spacecraft with respect to the some reference frame. Here, the satellite has called by body frame or rotating frame. Earth is an inertial reference frame it is fixed. It has called by non-rotating frame. [4].

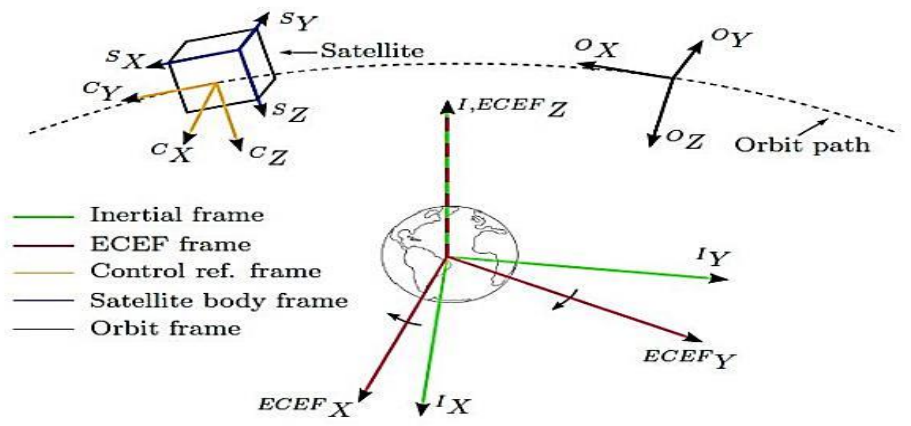

Fig. 3: Different Types of Coordinate Frame. 


\subsection{Geometrical definitions:}

The transformation (Figure: 3 ) from inertial reference (I, J, K) frame to the local orbital frame ( $\mathrm{x}_{0}, \mathrm{y}_{\mathrm{o}}, \mathrm{Z}_{\mathrm{o}}$ ) is performed by means of the Euler rotation sequence via the rotation angles $(\Omega, \mathrm{i}, \mathrm{u}) . \mathrm{u}$ stands for the latitude (argument) $\mathrm{u}=$ argument of perigee $(\grave{\omega})+$ true anomaly $(\theta)$. The angle of inclination has given by i, and vernal equinox with respect to the perigee has given by $\Omega$. [4]

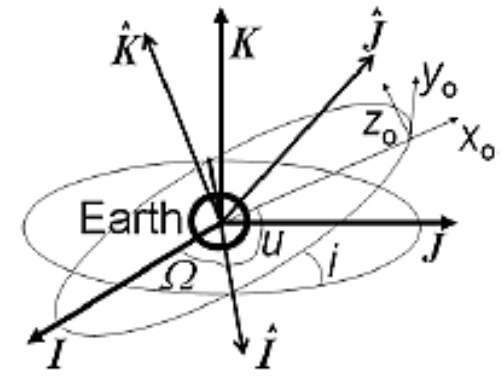

Fig. 4: Inertial Coordinate System (I, J, K) and Orbital Coordinate System.

The local orbital coordinate reference system (Figure 4) $\left(\mathrm{x}_{\mathrm{o}}, \mathrm{y}_{\mathrm{o}}, \mathrm{z}_{\mathrm{o}}\right)$ and the Earth Frame ( Satellite body frame attached with center of the body) (Reference Frame) ( $\mathrm{x}_{\mathrm{b}}, \mathrm{y}_{\mathrm{b}}, \mathrm{z}_{\mathrm{b}}$ ) The nominal attitude of RF (Figure 5) is along the orbital reference axes; $\mathrm{x}_{\mathrm{b}}=\mathrm{x}_{0}, \mathrm{y}_{\mathrm{b}}=\mathrm{y}_{\mathrm{o}}, \mathrm{z}_{\mathrm{b}}=\mathrm{z}_{\mathrm{o}}$. In this case, the yb axis is nominally along the velocity direction $\mathrm{V}$. [5].
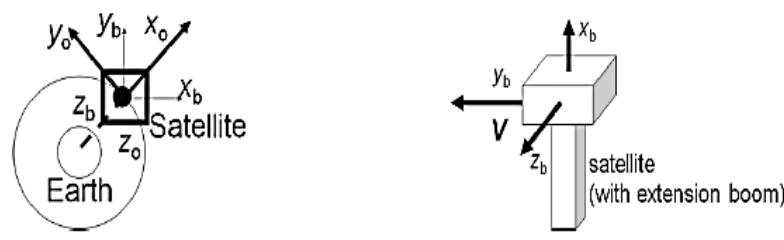

Fig. 5: Orbital Frame and Body Centered Reference Frame.

The series of transformations from the orbital coordinate system to the RF frame is 3-2-1 via the (yaw, pitch, Roll) angles. As shown in Figure 6, z-axis shows the rotation of yaw, y-axis shows the pitch rotation of pitch, and x-axis shows the rotation of roll.
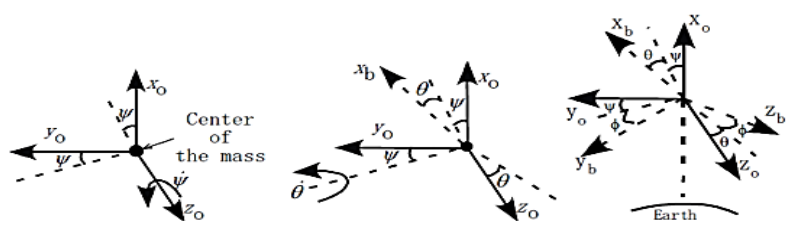

Fig. 6: Satellite Reference Frame to Orbital Reference Frame.

For the satellite: [6], [7]

Satellite rotates about the $\mathrm{x}_{\mathrm{o}}$ axis called as $\phi$ (Roll angle)

Satellite rotates about the $y_{0}$ axis called as $\theta$ (Pitch angle)

Satellite rotates about the $\mathrm{z}_{\mathrm{o}}$ axis called as $\psi$ (Yaw angle)

\section{Quaternions}

A quaternion is an efficient way to transform one frame (Body frame) to another frame (Inertial frame: Earth frame). It will not affect by singularity such as Euler angle. For visualizing the attitude of the satellite Euler angle is very useful tool. However, orientation of object in a 3D space quaternion more used to vector transform from body to inertial frame. Figure (7) Quaternion converts the rotation matrix and transform to another frame. It has four elements compare to the Euler transformation have nine element. Propagation of the satellite orientation calculates by quaternion. Transformation from body frame (satellite) to inertial frame quaternion method is more useful then Euler method. [8], [9].

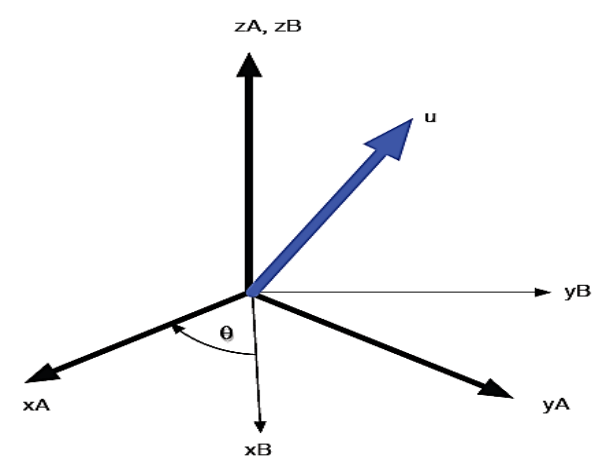

Fig. 7: Quaternion Diagram.

Quaternion ( $\mathrm{q}$ ) define as $\mathrm{q}=\left(\mathrm{q}_{1} \mathrm{w}+\mathrm{q}_{2} \mathrm{xi}+\mathrm{q}_{3} \mathrm{yj}+\mathrm{qz} 4 \mathrm{k}\right)$ It consist of four elements. 
It has both real and complex number coveting the vector from quaternion operation have 30 floating value and 45 processes to transform from a quaternion to a matrix. It was first define by William Rowan Hamilton. It avoids the singularity difficult in Euler angle transformation from one frame to another frame. The above diagram (Figure 7) discussing about two-reference frame $\left(\mathrm{x}_{\mathrm{A}}, \mathrm{y}_{\mathrm{A}}, \mathrm{z}_{\mathrm{A}}\right)$ and $\left(\mathrm{x}_{\mathrm{B}}, \mathrm{y}_{\mathrm{B}}, \mathrm{z}_{\mathrm{B}}\right)$. For satellite, rotate about $\mathrm{z}$-axis. In case, both vectors in both the frame are same but different value in $\mathrm{x}$ component and $\mathrm{y}$ component. A vector ' $u$ ' can be describe in any frame. For example, vector $u$ has same length it is significant to describe the quaternion. Quaternion frame 1 to frame 2 converts a vector. Frame 'A' vector denoted to Frame 'B' vector. Quaternion defines the magnitude and direction. [10]

\subsection{Rotation matrix}

The rotation matrix has defined as

$$
R^{i b}=\left[\begin{array}{lll}
1-2\left(q_{2}^{2}+q_{3}^{2}\right) & 2\left(q_{1} q_{2}+q_{3} q_{4}\right) & 2\left(q_{1} q_{3}-q_{2} q_{4}\right) \\
2\left(q_{2} q_{1}-q_{3} q_{4}\right) & 1-2\left(q_{1}^{2}+q_{3}^{2}\right) & 2\left(q_{2} q_{3}+q_{1} q_{4}\right) \\
2\left(q_{3} q_{1}+q_{2} q_{4}\right) & 2\left(q_{3} q_{2}-q_{1} q_{4}\right) & 1-2\left(q_{1}^{2}+q_{2}^{2}\right)
\end{array}\right]
$$

This same quaternion might be notated instead as qBA, where the order of the subscripts now indicates that the quaternion transforms a vector in frame A to a vector in frame B. [11]This form can be helpful when combining quaternions in order as discussed below. Both are use in practice and it is important to verify the rotation represented.

\section{Aerodynamic drag force}

Aerodynamic drag is predominant force affecting the satellite in the low earth orbit. When a satellite body moving in the atmosphere by GAS particle and liquid particle produces the resistance offered due to drag force. The drag force is more effects during launch the satellite and re-entry the space vehicle. When satellite is enter to the upper atmosphere because of the gravitational attraction and aerodynamic drag effect are consider more altitude between $100 \mathrm{~km}$ t0 $140 \mathrm{~km}$. Finally, to dis-integrate the satellite part and further enters to the atmosphere to decrease the orbital decay. To reduce the altitude because of these forces. Due to this effect, decrease the lifetime of the satellite. The drag force of the body is directly proportional to the square of the velocity and area of the satellite body. [12]

Aerodynamic drag force has given by

$$
F_{\text {drag }}=\frac{1}{2} \rho v^{2} A c_{d}
$$

Where, $F_{\text {drag }}$ is the aerodynamic drag force acting on the satellite $\rho$ is the density of atmosphere at that level

$v$ is velocity of satellite

A is frontal Area of Satellite,

$C_{d}$ is the coefficient of drag force

Coefficient of drag force is typically between 2 to 4 .

The altitude above $90 \mathrm{~km}$, Because of SUN effects extreme ultraviolet radiation and more temperature with respect to altitude. And altitude $200 \mathrm{~km}$ to $260 \mathrm{~km}$ temperature is reduces to $600 \mathrm{~K}$ to $1150 \mathrm{~K}$ and because of the solar activity high density. Because of the solar radiation satellite will, decay in maximum more solar radiation affects the satellite surface and minimum decay during minimal solar perturbation. For circular orbit the changes in a, $\mathrm{T}, \mathrm{v}$ per revolution as given equation (11)

$$
\begin{gathered}
\Delta_{a_{\text {rev }}}=-\frac{2 \pi C_{d} A \rho a^{2}}{m} \\
\Delta P_{\text {rev }}=-\frac{6 \pi^{2} C_{d} A \rho a^{2}}{m V} \\
\Delta V_{\text {rev }}=\frac{\pi C_{d} A \rho a V}{m} \\
a_{r}=-\frac{4.5 \times 10^{-8} A}{m}
\end{gathered}
$$

Ballistic coefficient is depends upon the mass of the satellite and area of the satellite it is described by $m /\left(C_{d} A\right)$, It is constant for most of the satellite. Drag effects is more when low valve of ballistic constant and Drag is less when high value of ballistic constant.

Hubble Space Telescope has initially designed to maintain pointing accuracy of at least 7/1000th of an arc second. This dependent on disturbance effects accumulating over time. Disturbances acting upon a satellite can divided into external and internal. External effects are those characterizing the Space environment. They would act event if a spacecraft itself a rigid body. Internal disturbances are closely tied witch spacecraft structure, in particular: internal moving parts and mass or radiation has emitted. [12] 


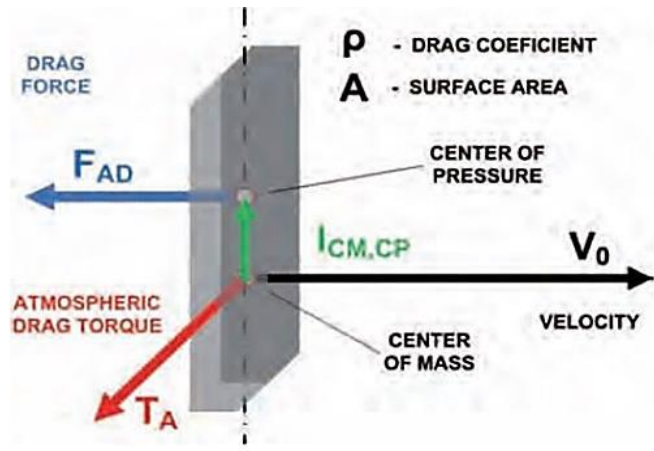

Fig. 8: Aerodynamic Drag.

To estimate the life time of the satellite life cycle (Eqn. 12) as given equation

$$
L=\frac{-H}{\Delta a_{\text {rev }}}
$$

The above equation describes the changes in atmospheric density due to altitude variation and solar changes. $\mathrm{H}$ is mean by atmospheric scale height of density.

\section{Solar perturbation}

The Solar perturbation (Eqn. 13) is periodic variation in the orbital components; solar radiation force as given by [13]

$$
a_{r}=-\frac{4.5 \times 10^{-8} A}{m}
$$

A is defined by cross section area of the satellite surface visible to the sun $\mathrm{m}$ is defined by mass of the satellite, $\mathrm{kg}$

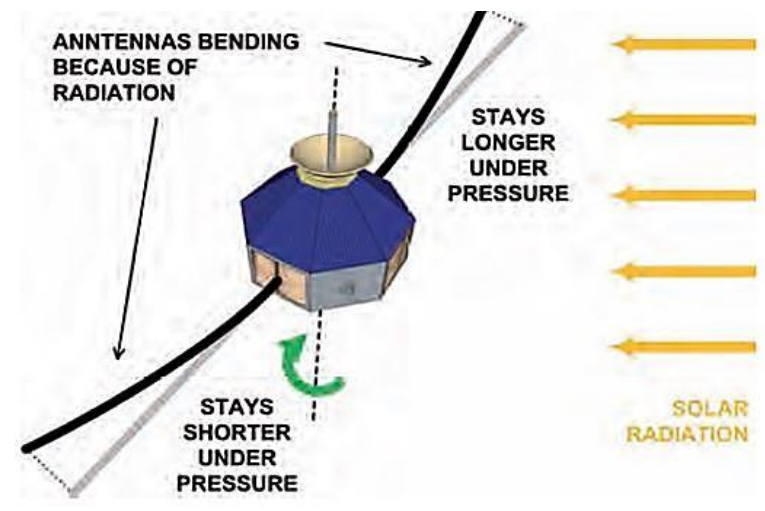

Fig. 9: Solar Radiation.

For altitude, $800 \mathrm{~km}$ below have more aerodynamic drag acceleration and less solar radiation force or pressure. In addition, above $800 \mathrm{~km}$ solar radiation pressure (Figure 9) is greater than the aerodynamic drag force. In this region predominant effects of perturbation due to the solar activity. [13]

\section{Attitude control with control momentum gyroscope}

The Attitude Control of satellite with control momentum gyroscope attached with stable platform. It has called as Gimbal (single gimbal or Dual gimbal). [14] When design the attitude control technique. Pitch control with momentum wheel as shown in the Figure (10)

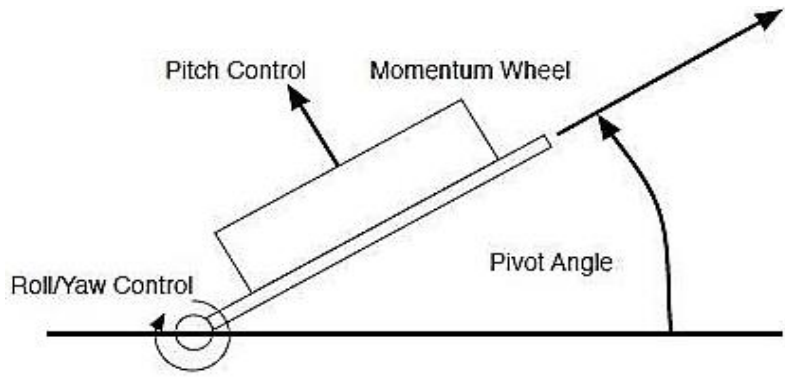

Fig. 10: Position of the Momentum Wheels.

It is very important to analysis the low weight and low cost method. [15] Single gimbal variable speed control momentum gyroscope, (SGVSCMG) have overcome the cost and weight consideration. 


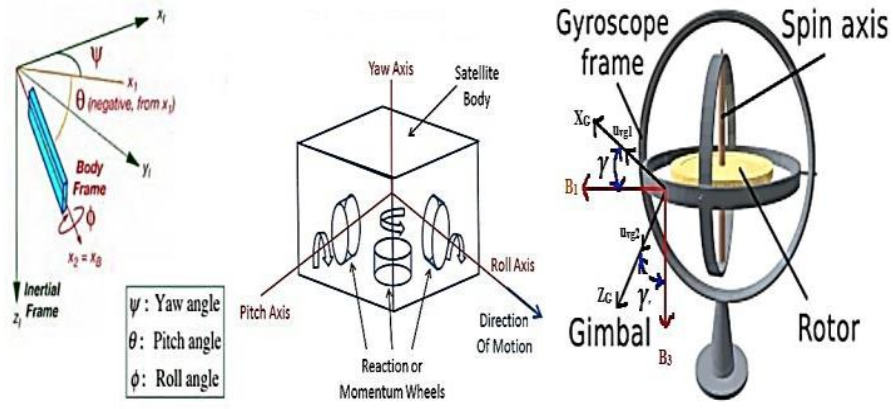

Fig. 11: Definition of Three Coordinates Frames with Gyroscope Frame.

\subsection{Angular momentum of the satellite}

The quaternion parameter represents the attitude orientation of the satellite. These parameters have one real and three imaginary quantities as describe below. Quaternion parameter relate with the angular frequency defined by [15]

$$
\text { quaternion }=\llbracket \frac{q 0}{q v} \rrbracket=\llbracket \frac{\frac{q 0}{q x}}{\frac{q y}{q z}} \rrbracket
$$

$\llbracket \frac{\frac{q 0}{q 1}}{\frac{q 2}{q 3}} \rrbracket$

$0.5 *[$ quaternion parameters $] *[$ angular velacity $]$

Where q0, qx, qy, and qz are the parameters quaternion, and $\omega=[\omega 1, \omega 2, \omega 3]^{\mathrm{T}}$ are angular speed vector of satellite about the satellite body reference coordinates

$$
\begin{gathered}
C v_{\text {Body }}^{\text {Inertial }}=\llbracket C v_{j k} \rrbracket \\
\mathrm{j}, \mathrm{k}=1,2,3 \\
C_{\text {Body }}^{\text {Inertial }}=C_{B}^{I} \\
\mathrm{Cvj \textrm {k }}=\cos * \beta \mathrm{jk} \\
C v_{\text {Body }}^{\text {Inertıal }}=C v_{B}^{I}=[0 \omega 3 \omega 2 ; \omega 30-\omega 1 ;-\omega 2 \omega 10]
\end{gathered}
$$

The Direct cosine matrix included in the quaternion because it is easy way to describe the axis of satellite frame. [15]Control the $Z_{B}$ direction the DCM is describe by $C v_{B o d y}^{\text {Inertial }}$ equation given by (Figure 11)

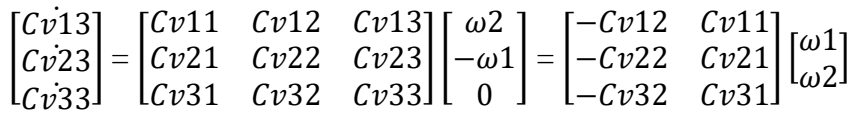

The net angular momentum (Eqn. 14) in the satellite is not equal to zero. It is constant w.r.t fixed reference frame

$$
\mathrm{h}_{\text {momentum }}=\mathrm{J} \omega+J_{G(\text { Gimbal })} \dot{\gamma} g_{2}+J_{W(\text { Wheel })} \Omega g_{3} \neq 0
$$

Where $\mathrm{J}=\operatorname{diag}[\mathrm{J} 1 ; \mathrm{J} 2 ; \mathrm{J} 3]$ satellite inertia matrix with gyroscope in the inner gimbal and wheel attached with it. [16] Wheel and Gimbal are denoted as $\mathrm{J}_{\text {Wheelis }}$ the M.O.I of the wheel defecting about $\mathrm{Z}_{\mathrm{G}}$ axis and $\mathrm{J}_{\text {Gimbalis }}$ the M.O.I of gimbal rotation about $\mathrm{Y}_{\mathrm{G}}$. Revolution of the Wheel is $\Omega$

M.O.I is moment of inertia

The equation of motion of the satellite as Angular momentum,

$$
\begin{aligned}
(\mathrm{h})=\left[\begin{array}{l}
\text { hmoment } 1 \\
\text { hmoment } 2 \\
\text { hmoment } 3
\end{array}\right] & =\left[\begin{array}{c}
J 1 \omega 1+J_{W(\text { Wheel })} \Omega \sin \gamma \\
j 2 \omega 2 \\
J 3 \omega 3+J_{W(\text { Wheel })} \Omega \cos \gamma
\end{array}\right] \\
& \dot{h}+\omega \times \mathrm{h}
\end{aligned}
$$

The angle of gimbal is $\gamma$ and acceleration of wheel is $\dot{\Omega}$ both are used to produces the feedback torque, that need to control the actuator input

$$
\dot{\gamma}=u 1, \quad \dot{\Omega}=u 2
$$




\subsection{Motion equation of satellite in the body frame}

$$
\left[\begin{array}{l}
v 1 \\
v 3
\end{array}\right]=\left[\begin{array}{cc}
-\Omega * C * \gamma & -S * \gamma \\
\Omega * S * V & -C * \gamma
\end{array}\right]\left[\begin{array}{l}
u 1 \\
u 2
\end{array}\right]
$$

$\mathrm{C}=\cos , \mathrm{S}=\sin$

This dynamic equation of the satellite will used to design the control technique of the model

\section{Gradient (GRAVITY) torque: (GT)}

Assumptions: Upper-order relations ignored.

Rigid Satellite body assumed. [16]

$$
\mathrm{T}_{\text {gravity gradient }}=3\left[\left(\mathrm{I}_{3}-\mathrm{I}_{2}\right) \mathrm{m} \mathrm{n} \mathrm{i}+\left(\mathrm{I}_{1}-\mathrm{I}_{3}\right) \ln \mathrm{i}+\left(\mathrm{I}_{2}-\mathrm{I}_{1}\right) l \mathrm{~m} \mathrm{k}\right]_{\dot{\theta}} 2 /(1+\mathrm{e} \cos \theta \mathrm{v})
$$

Variables: $\mathrm{T}$ gravity gradient $=$ gravity gradient torque

$\theta=$ true anomaly

$\mathrm{e}=$ orbital eccentricity

$\mathrm{I}_{1}, \mathrm{I}_{2}, \mathrm{I}_{3}=$ moments of inertia of principle axis of body

$\mathrm{l}, \mathrm{m}, \mathrm{n},=$ (satellite with principle axis of body) - direction cosines

\subsection{Gravity gradient stabilization}

Gravity gradient torque disappears:

- Angle between 2 vectors, cosine are zero.

- $\quad \mathrm{I} 1=\mathrm{I} 2=\mathrm{I} 3$

- In the axis one cosine are zero other two moment of inertia equal

Gravity gradient torque ( $\left.\mathrm{T}_{\text {gravity }}\right)$ is a perturbation in the orbit. When maximum moment of inactivity in the orbit plane \& minimum moment of inactivity in the local frame this configuration to orient the stabilized satellite. [15]

Gravity gradient means a small mass injected from the satellite body for desaturation method to stabilize the attitude of body. This method has used for low earth orbit. For high earth orbit like geostationary Earth, orbit (GEO) it has less accuracy to stabilize the satellite. It needs the more effective damping elements.

The eccentricity decides the size of the orbit. Gravity gradient torque affects the eccentricity of the elliptical orbit. Periodically changes the position of the satellite.

\section{Magnetic torquer (di-pole)}

Magnetic Torquers generate the magnetic flux, which reacts with earth magnetic flux to control the two axis of the satellite. For three axis control, (CMG) Control momentum gyro are used for stabilize the satellite. Low earth orbit magnetic thruster reduces the disturbance of earth magnetic effect. The Earth magnetic field measured by magnetometer. Periodic disturbance controlled by momentum wheel (or) Flywheels. Satellite Carrie Bar magnet with coil reacts the Di-pole moment. Torque on a di-pole is uniform or constant magnetic field torque required keeping the dipole perpendicular to the constant magnetic field. Dipole moment is product of strength of pole separated by two plates. It has North Pole (N) and South Pole (S) both force have equal magnitude and opposite direction. Magnetic dipole moment (MDM) expressed in NM/T

$$
\mathrm{T}=\mathrm{M} * \mathrm{~B}
$$

Magnetic field (B) and dipole (M) of the torque. For an air armature (or) conductor, the dipole is

$$
\mathrm{M}=\mathrm{N} * \mathrm{I} * \mathrm{~A}
$$

Where Coil (or) Winding no of turns is N, the current in the coil is I, and, the area of the winding is A, Characteristically, coil is wounded from place to place of the satellite structure or body.

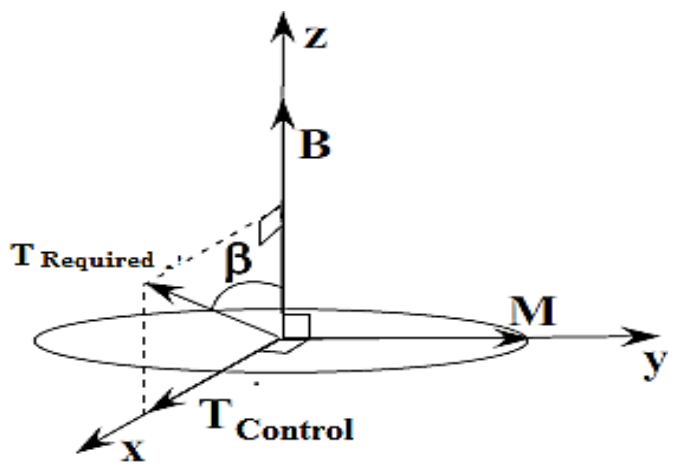

Fig. 12: Regulator Torque $T_{R}$ and Magnetic Di-Pole Moment (MDM). 
From the Figure 12. Calculating the magnetic moments, required control torque, $\mathrm{T}_{\text {Required }}$ and magnetic flux vector, $\mathrm{B}$ in the satellite frame from the magnetometer. [15], [16] Introduce control torque, Tcontrol which we can actually be generated by the magnetic torquers. Because Tcontrol must be regular to the magnetic flux, we have $\mathrm{BT}$ control $=0$. Most efficient magnetic moment is the one that always points normal to the B vector, so that MTcontrol = zero. Control torque can calculates from Magnetic field (B) and Regulated torque ( $\mathrm{T}_{\text {Required}}$ ). Magnetic torquer produce the torque in the low earth orbit altitude of $1000 \mathrm{KM}$ generate $0.05 \mathrm{~N}-\mathrm{M}$

The respective unit vectors are defined as: $\left(\mathrm{e}_{\mathrm{x}}, \mathrm{e}_{\mathrm{y}}, \mathrm{e}_{\mathrm{z}}\right)=\left(\right.$ Tcontrol $\left./ \mathrm{T}_{\text {control, }} \mathrm{M} / \mathrm{M}, \mathrm{B} / \mathrm{B}\right)$

Hence, the Control torque vector is: (Tc)

Tcontrol $=\left(\mathrm{T}_{\text {Required }} * \mathrm{~s} * \beta_{\text {plashma }}\right) \mathrm{e}_{\mathrm{x}}=\mathrm{T}_{\text {Required }}-\left(\mathrm{T}_{\text {Required }} * \mathrm{c} * \beta_{\text {plashm }}\right) \mathrm{e}_{\mathrm{z}}=\mathrm{T}_{\text {Required }}-\left(\mathrm{T}_{\text {Required }} * \mathrm{c} * \beta_{\text {plashm }}\right) \mathrm{B} / \mathrm{B}$

$\mathrm{s}=\sin$ and $\mathrm{c}=\cos$ terms

$$
\mathrm{T}_{\mathrm{control}}=\mathrm{M} * \mathrm{~B}
$$

MDM is the magnetic dipole or solenoid moment, $\mathrm{M}$ well defined by pole strength and distance between two magnetic poles, $\mathrm{M}$ is inversely proportional to the Magnetic field B and Required torque vectors $\mathrm{T}_{\text {Required }}$

$\mathrm{M} \alpha \mathrm{B} . \mathrm{T}_{\text {Required }}$

$$
\mathrm{M}=\frac{B \cdot \text { TRequired }}{B^{2}}
$$

Nadir is mean by satellite looking as string line to the earth surface; Satellite and earth have same line of sight. [16] Zenith exactly opposite to the Nadir the satellite moment around Nadir/Zenith direction has expressed by Yaw angle and velocity direction has expressed by Yaw Roll, orbit direction (or) movement is expressed by Pitch Roll

\subsection{Solenoids}

Solenoids are bar magnet and current caring conductor or coil creates the Di-Pole moment. It is an electromagnetic armature used in satellite system. Interaction between earth magnetic flux vs. Magnetic torquer derives this section.

\subsection{Motion equation (EOM) for twin coil BAR magnet and coil}

In free space, Magnetic field plays major role in the permeability. And if we consider closed surface area or nozzle area permeability of median it is assumed as infinity, Therefore magnetic field are consider zero $(=0)$

$$
\oint_{C}^{1} \text { Magnetic field . } \mathrm{dl}=\int_{S}^{1} J n d a
$$

Above equation, explain about if we integrate the magnetic field in the closed path in the surface area S. It is equal to the integral of all the Current (I) in the surface in the path

\section{State prediction with unscented Kalman filter (UKF)}

The Extended Kalman Filter (EKF) is use for estimate and predicts the state (or) information from the attitude sensor in the linear system. Extended KF is more efficiency and accurate method to predict the performance in the non-linear system. Hence, the unscented transformation (UT) occurs in sate equation. First and second order ordinary differential equation (ODE) in the non-linear system estimated by UKF. The EKF and UKF are unlike for derivatives or Jacobians in the estimate the performance equation and state equation. [17] It requires propagating state covariance matrix, Filter circulates state, and standard deviations has circulated. This indicates the random changes in the state or uncertainty. [18]

Parameter estimators \& classifications of weights:

Weight for the state and estimate the parameter

$$
\text { Weight }=W_{0}^{m}=\frac{\text { state }}{\text { measuement }+ \text { state }}
$$

Prepare the filter factor with the expected value of the parameters

State $\left(\mathrm{t}_{\mathrm{o}}\right)=$ Expected Value $\left\{\mathrm{x}_{0}\right\}$

$\mathrm{x}_{0}=$ Initial state parameter

Covariance for the parameters

$\mathrm{px}_{0}=$ Expected Value $\left\{\left(\right.\right.$ state $\left.\left.\left(\mathrm{t}_{\mathrm{o}}\right)-\mathrm{x}_{\mathrm{o}}\right)\left(\text { state }\left(\mathrm{t}_{\mathrm{o}}\right)-\mathrm{x}_{\mathrm{o}}\right)^{\mathrm{T}}\right\}$

The standard deviations are calculated. Initial parameter for the estimation by including the $\sqrt{\text { covariance matrix }}$ for the estimator of parameter.

Equation for the state:

$\mathrm{X}_{\text {initial }}=$ function of $\mathrm{xi}, \mathrm{u}, \mathrm{w}$, and $\mathrm{t}$ 
Covariance form for the state:

$$
=\sum_{t=0}^{2 L} W_{i}^{C}(x i-x)(x i-x) T
$$

Measurements (Expected):

Output $=$ h (state $)$

Mean measurement: $=\sum_{t=0}^{2 L} W_{i}^{M} y i$

The covariance's are

The Kalman gain $\left(\mathrm{K}_{\text {Gain }}\right): \mathrm{K}_{\text {Gain }}=\mathrm{P}_{\mathrm{xy}} \mathrm{p}_{\mathrm{yy}}{ }^{-1}$

$$
\begin{aligned}
& \mathrm{P}_{\mathrm{yy}}=\mathrm{y}=\sum_{t=0}^{2 L} W_{i}^{C}(y i-y)(y i-y) T \\
& \mathrm{P}_{\mathrm{xy}}=\mathrm{y}=\sum_{t=0}^{2 L} W_{i}^{C}(x i-x)(y i-y) T
\end{aligned}
$$

The state update is

$\mathrm{X}=$ state + Kalman Gain $(\mathrm{K})($ Actual measurement $(\mathrm{y})-\dot{y})$

Covariance updates $\left(\mathrm{P}_{\mathrm{CU}}\right): \quad \mathrm{P}_{\mathrm{CU}}=\mathrm{P}-\mathrm{K}_{\mathrm{Gain}} \mathrm{P}_{\mathrm{yy}} \mathrm{K}^{\mathrm{T}}$

' $y$ ' is the actual measurement matches the time for updated state.

The update classification starts by including the constraint design random sequence of covariance or uncertainty (Quncer)

$$
\mathrm{P}_{\mathrm{CU}}=\mathrm{P}+\text { Quncer }
$$

The parameter update:

$$
\mathrm{w}=\mathrm{w}+\mathrm{K}(\mathrm{y}-\dot{y})
$$

Covariance updates $\left(\mathrm{P}_{\mathrm{CU}}\right)$ :

$$
\mathrm{P}_{\mathrm{CU}}=\mathrm{P}-\mathrm{K}_{\text {Gain }} \mathrm{P}_{\mathrm{yy}} \mathrm{KT}
$$

' $y$ ' is the actual measurement matches the time for updated state.

Hence, nonlinear state equations are $\quad \dot{r}=\mathrm{v}$

$$
\dot{v}+\frac{\text { Gravitational Parameter } * r}{\left(r^{T} r\right) 3 / 2}+\sum_{K} a k=\frac{f}{m}
$$

$\mathrm{m}$ is the spacecraft mass. Position vector, r. solar pressure force on the spacecraft, f. [19],[20]

\section{Simulation result}

Effect of solar radiation on satellite

Area of satellite facing the sun decreases to a certain limit (threshold limit) with respect to the decrease in deceleration due to solar radiation and after the threshold limit it increase. Mass of satellite facing the sun decreases to a certain limit (threshold limit) with respect to the decrease in deceleration due to solar radiation and after the threshold limit, it increases.

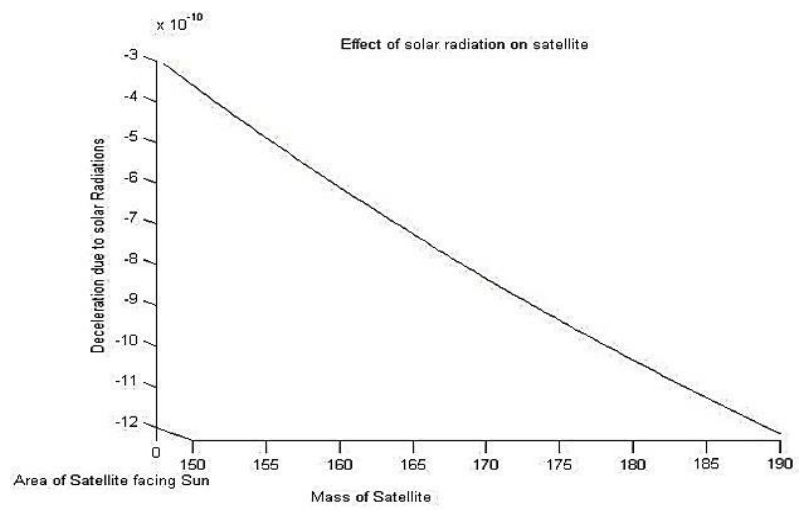

Fig. 13: Effect of Solar Radiation on Satellite Mass v/s Deceleration.

Effect of atmospheric drag

This graph is hyperbolic in nature. Ballistic constant increases with the decrease in acceleration due to atmospheric drag. 
- Change in velocity per revolution due to atmospheric drag vs ballistic constant

This graph has a logarithmic decrement kind of nature. Ballistic constant increases with the decrease in change in velocity per revolution due to atmospheric drag.

- Lifetime of satellite in second's vs deceleration due to atmospheric drag

The lifetime of satellites decreases with the decrease in deceleration due to atmospheric drag

- Change in revolution period due to atmospheric vs ballistic constant. This graph is hyperbolic in nature. Ballistic constant increases with decrease in change in revolution period due to atmospheric drag.

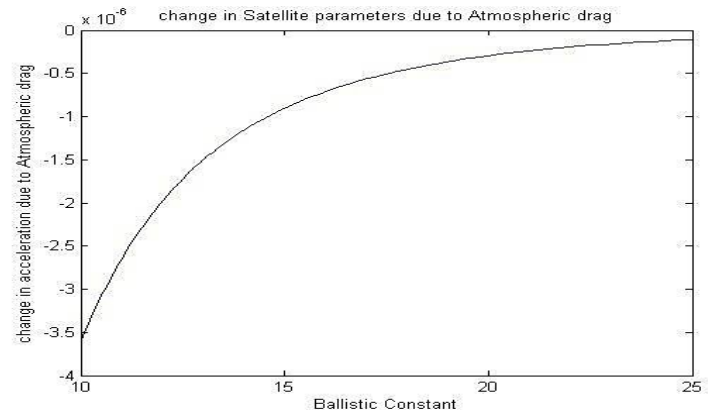

Fig. 14: Change in satellite semi major Axis

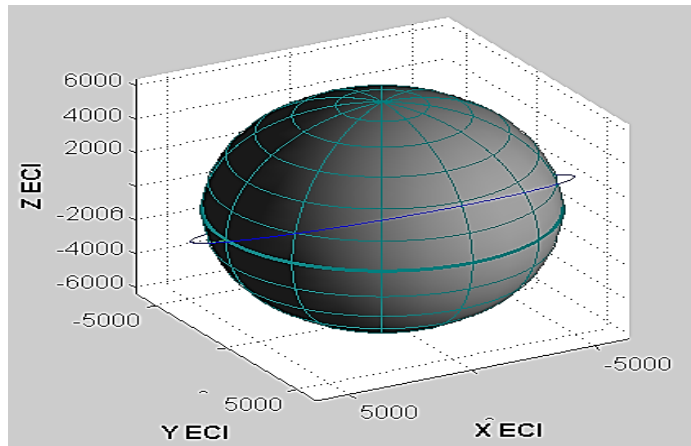

Fig. 15: Earth Orbit.

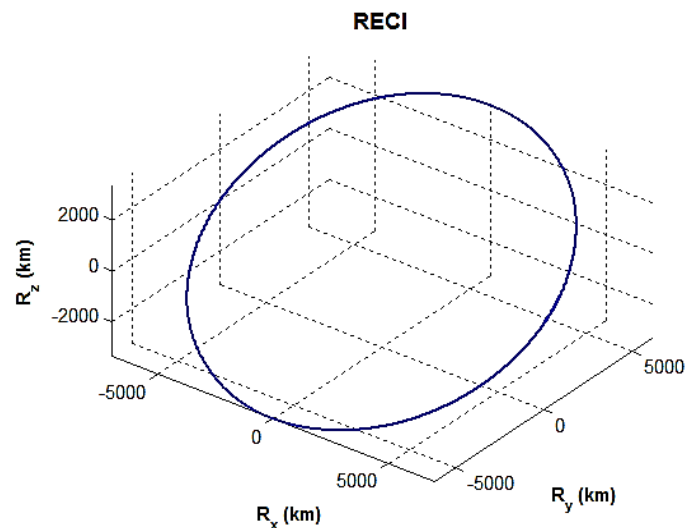

Fig. 16: ECI Frame

The above diagram ECI Frame describing the Six DOF simulation with point mass orbit, rigid body dynamics.

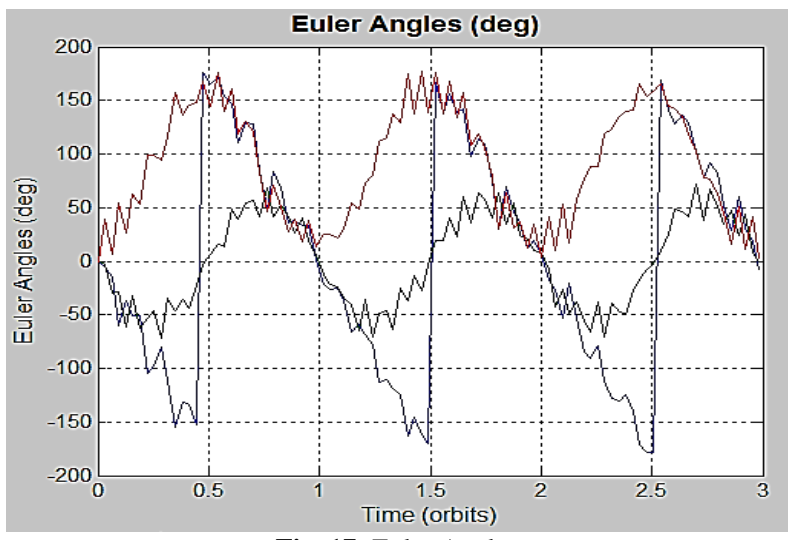

Fig. 17: Euler Angles. 


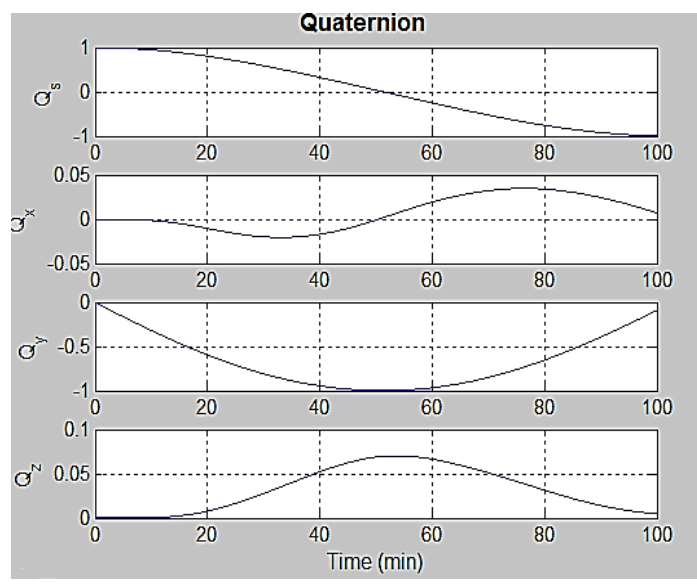

Fig. 18: Quaternion.

Variation in the Euler angles (Shown in Figure 17) in Orbit due to the disturbances, the Low earth orbit because of aerodynamic drag, solar pressure, Gravitational attraction (Non Spherical of earth surface). Quaternion (Figure 18) representing the attitude of satellite.
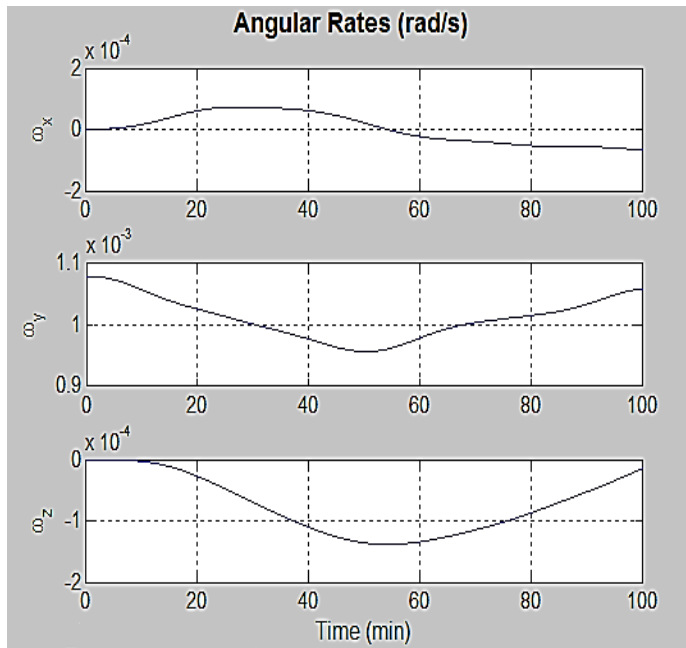

Fig. 19: Angular Rate.

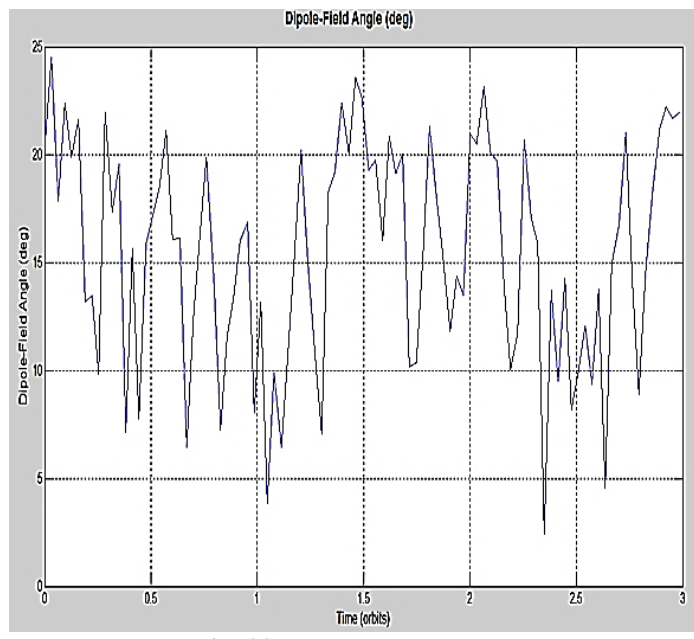

Fig. 20: Dipole-Field Angle.

Variation in angular rate due to orbit in the satellite because interaction of earth magnet combine with the magnetic torques (or) BAR Magnet (Earth is a giant magnet) with coil (or) conductor. (Figure 20). 


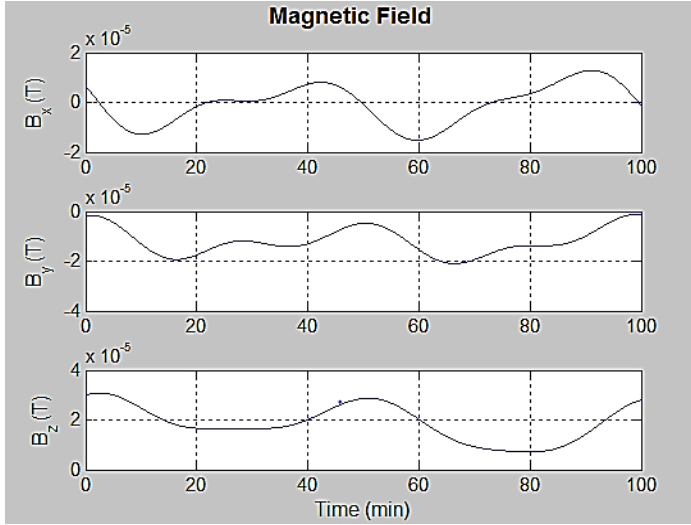

Fig. 21: Magnetic Field.

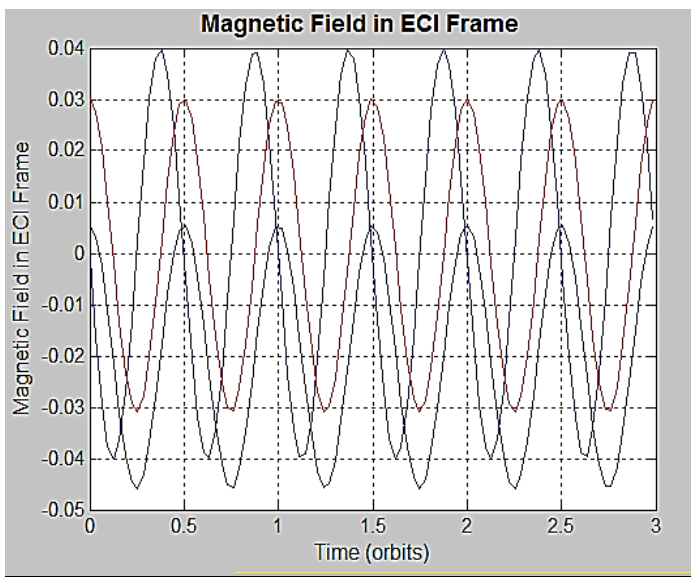

Fig. 22: Magnetic Field in ECI Frame.

Magnetic torque handled explicitly in the RHS Satellite has modeled with magnetic torques in ECI Frame. Magnetic field ( $\left.\mathrm{B}_{\mathrm{x}}, \mathrm{B}_{\mathrm{y}}, \mathrm{B}_{\mathrm{z}}\right)$ shown in Figure 21. The magnetic field in Earth centered inertial (ECI) frame as shown in Figure 22. Variation in the magnetic field due to the surface of the earth. Non-homogeneity of earth produces the $\mathrm{J}_{2}$ Perturbation.

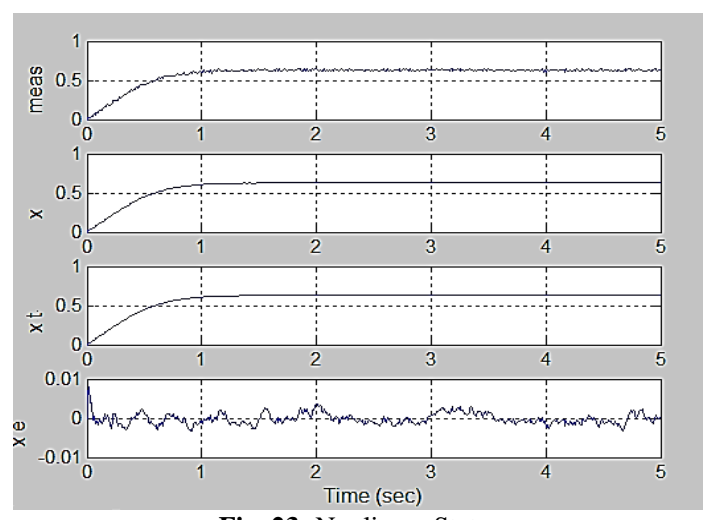

Fig. 23: Nonlinear State.

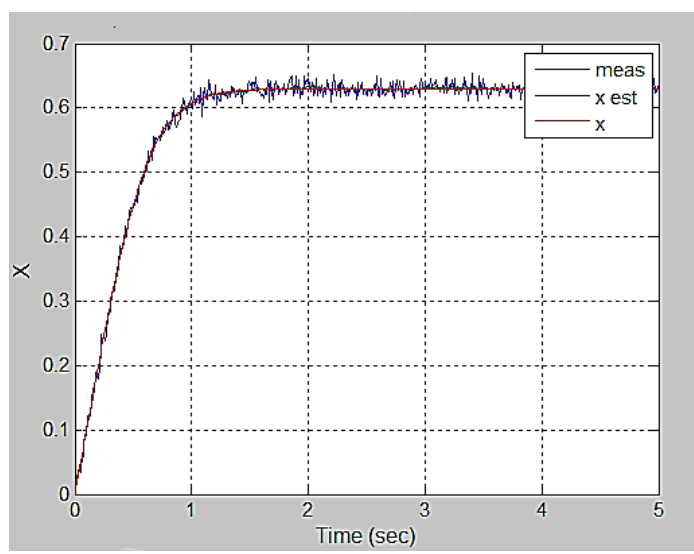

Fig. 24: Nonlinear Measurement.

The simulation shows the measurement vs. estimation of the state. 


\section{Results}

This research is focus to control the attitude of low earth orbiting satellite less than 1000KM altitude. The experimentally analyses for interaction between earth magnetic field and magnetic torquer or di-pole moment create the control torque around the satellite body was derived. Also, discuss the perturbation in the low earth orbit (LEO) such as Aerodynamic drag, solar force. Attitude of the satellite for low earth orbit discussed with the help of quaternion and Euler angle.

\section{Conclusions}

The paper presents, the position and orientation of satellite attitude quaternion has derived. It is very accurate tool compare with Euler angle method. Singularity problems has omitted in quaternion attitude estimation techniques because it has four elements only. In case of Euler method three axis (3X3) nine elements. Variation of angular rate of the body axis due to orbital perturbation in the low earth orbit (such as Aerodynamic drag, Gravitational attraction of the earth, solar pressure, and earth magnetics field is performed. The calculation of attitude control system using magnetic torque has discussed. The error estimation using of unscented Kalman filter obtained by MATLAB Simulation.

\section{References}

[1] Perry, W.R. (1967). "Orbital Mechanics". In Theodore Baumeister. Marks' Standard Handbook for Mechanical Engineers (Seventh ed.). New York City: McGraw Hill. pp. 11:151-52. ISBN 0-07-142867-4.

[2] Sidi, M.J. "Spacecraft Dynamics \& Control. Cambridge, 1997. https://doi.org/10.1017/CBO9780511815652.

[3] Thomson, W.T. "Introduction to Space Dynamics." Dover, 1961.

[4] Beer, Ferdinand P.; Johnston, Russell, Jr. (1972), Vector Mechanics for Engineers: Statics \& Dynamics, McGraw-Hill

[5] Kromis, A.J. (1967). "Powered-Flight-Trajectory Analysis". In Theodore Baumeister. Marks' Standard Handbook for Mechanical Engineers (Seventh ed.). New York City: McGraw Hill. pp. 11:154-55. ISBN 0-07-142867-4.

[6] Wertz, J.R. "Spacecraft Attitude Determination and Control." Kluwer, 1978. https://doi.org/10.1007/978-94-009-9907-7.

[7] Anderson, John D. (2004), Introduction to Flight (5th ed.), McGraw-Hill, ISBN 0-07-282569-3

[8] Schaub, H., and Junkins, J. L., "MATLAB Toolbox for Rigid Body Kinematics," Proceedings of the AAS/AIAA Space Flight Mechanics Meeting, Breckenridge, CO, American Astronautical Soc. Paper 99-139, Springfield, VA, 7-10 Feb. 1999, pp. 549-560.

[9] Chaturvedi, N. A., Global Dynamics and Stabilization of Rigid Body Attitude Systems, Ph.D. Dissertation, Univ. of Michigan, Ann Arbor, MI, 2007.

[10] Jack B. Kuipers. Quaternions and Rotation Sequences. Princeton University Pres, 2002.

[11] Blanke, Mogens; Larsen, Martin Birkelund, Satellite Dynamics and Control in a Quaternion Formulation.

[12] Fellenz, D.W. (1967). "Atmospheric Entry". In Theodore Baumeister. Marks' Standard Handbook for Mechanical Engineers (Seventh ed.). New York City: McGraw Hill. pp. 11:155-58. ISBN 0-07-142867-4.

[13] van der Ha, J. C., and Lappas, V. J., "Long-Term Attitude Drift of Spinning Spacecraft Under Solar Radiation Torques,” Journal of Guidance, Control, and Dynamics,

[14] A. Craig Stickler, K. T. Alfriend, "Elementary Magnetic Attitude Control System." Journal of Spacecraft and Rockets, Vol. 13, No.5, 1976, pp. 282 https://doi.org/10.2514/3.57089.

[15] Mark .L. Psiaki, "Magnetic Torquer Attitude Control via Asymptotic Periodic Linear Quadratic Regulation.", Journal of Guidance, Control, and Dynamics, Vol. 24, No.2, 2001 https://doi.org/10.2514/2.4723.

[16] Levesque, J., "Passive Magnetic Attitude Stabilization using Hysteresis Materials.", 17th AIAA/USU Conference on Small Satellites, 2003.

[17] Markley, F. L., "Attitude Error Representation" Journal of Guidance, Control, and Dynamics, Vol. 26, No. 2, 2003 , pp. 311-317. https://doi.org/10.2514/2.5048.

[18] Julier, S. J. and Uhlmann, J. K. [1997], 'A new extension of the kalman filter to nonlinear systems. https://doi.org/10.1117/12.280797.

[19] Jiang, X.-Y. [2005], 'Unscented kalman filter for spacecraft attitude estimation and calibration using magnetometer measurements' Proceedings of the Fourth International Conference on Machine Learning and Cybernetics.

[20] VanDyke, M. C., Schwartz, J. L., and Hall, C. D., "Unscented Kalman Filtering for Spacecraft Attitude State and Parameter Estimation," Proceedings of the AAS/AIAA Space Flight Mechanics Conference, AAS Paper 2004-115, Maui, Hawaii, 8-12 Feb. 2004. 